Document downloaded from:

http://hdl.handle.net/10251/156323

This paper must be cited as:

Aragones, V.; Pérez De Castro, AM.; Cordero, T.; Cebolla Cornejo, J.; López Del Rincón, C.; Picó Sirvent, MB.; Daros Arnau, JA. (2019). A Watermelon mosaic virus clone tagged with the yellow visual maker phytoene synthase facilitates scoring infectivity in melon breeding programs. European Journal of Plant Pathology. 153:317-323.

https://doi.org/10.1007/s10658-018-01621-x

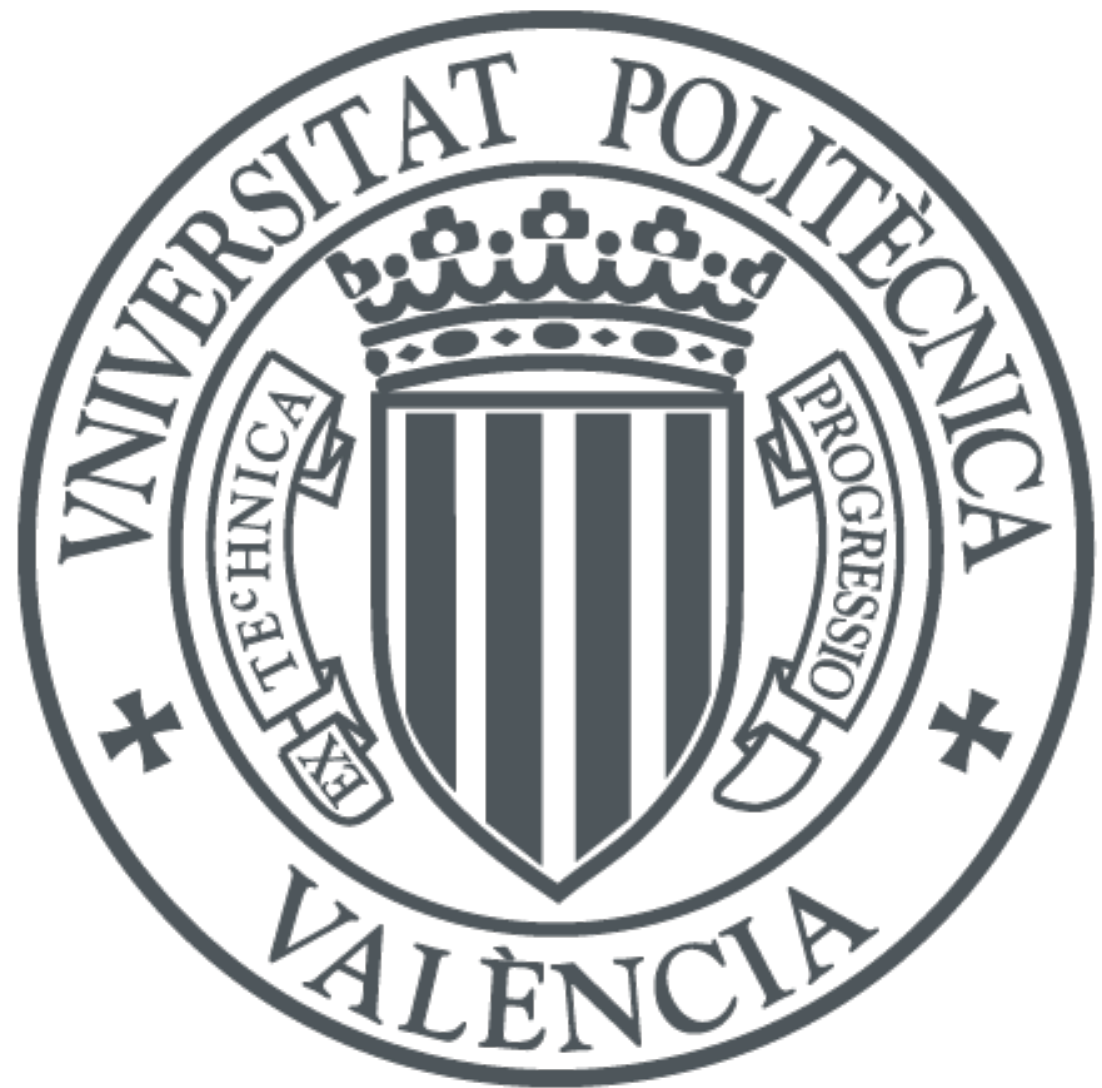

The final publication is available at

https://doi.org/10.1007/s10658-018-01621-x

Copyright Springer-Verlag

Additional Information 


\title{
A Watermelon mosaic virus clone tagged with the yellow visual maker phytoene synthase facilitates scoring infectivity in melon breeding programs
}

\author{
Verónica Aragonés, Ana Pérez-de-Castro, Teresa Cordero, Jaime Cebolla-Cornejo, \\ Carmelo López, Belén Picó, José-Antonio Daròs
}

\section{Aragonés, T. Cordero, J.-A. Daròs (*)}

Instituto de Biología Molecular y Celular de Plantas (Consejo Superior de Investigaciones Científicas-Universitat Politècnica de Valencia), Avenida de los Naranjos s/n, 46022 Valencia, Spain

e-mail: jadaros@ibmcp.upv.es

\author{
A. Pérez-de-Castro, J. Cebolla-Cornejo, C. López, B. Picó \\ Instituto de Conservación y Mejora de la Agrodiversidad Valenciana, Universitat Politècnica \\ de Valencia, 46022 Valencia, Spain
}

\begin{abstract}
Watermelon mosaic virus (WMV), a plus-strand RNA virus that belongs to the family Potyviridae, is one of the most damaging viruses that infect cucurbit crops. However, isolates of this species frequently induce mild symptoms, which makes difficult to manage the disease in the field and breeding programs. A new generation of marker genes has been recently developed to visually track plant virus infection. Virus-mediated expression of the Pantoea ananatis phytoene synthase (crtB) induces accumulation in infected tissues of pigmented carotenoids that can be readily detected by the naked eye. Here we investigated whether this visual marker may facilitate visual diagnosis of WMV infection in cucurbits. First, we cloned a mild WMV isolate (WMV-Vera) and built a recombinant clone that expresses crtB (WMV-crtB). Next, we inoculated a series of cucurbit cultivars frequently used in breeding programs. We observed that WMV-mediated expression of crtB helps to visually score infection in two susceptible and highly appreciated melon cultivars, such as Piñonet Piel de Sapo and Vedrantais, but not in susceptible Cucurbita spp. cultivars. An increase in yellow pigmentation was experimentally confirmed in susceptible melon cultivars
\end{abstract}


by measuring the Hue angle using a solid colorimeter. Carotenoid analyses in infected tissues explained why the visual crtB marker performs better in susceptible melon than in Cucurbita spp. cultivars. These results support that the visual marker crtB may facilitate visual diagnosis of WMV infection in melon breeding programs.

Keywords: Watermelon mosaic virus, phytoene synthase, melon, Cucurbita, virus diagnosis 
Watermelon mosaic virus (WMV) is one of the most damaging viruses that infect cucurbit crops worldwide. This is a plus-strand RNA virus that belongs to the genus Potyvirus within the family Potyviridae, which is efficiently transmitted by aphids in a non-persistent manner and presents a relatively broad host range (Desbiez and Lecoq 2004; Ouibrahim et al. 2014). As in all potyviruses (genus Potyvirus), WMV genome is an RNA molecule of about 10,000 nt that is covalently attached at the $5^{\prime}$ end to a viral genome-linked protein ( $\mathrm{VPg}$ ) and is polyadenylated at the 3' end. WMV RNA is encapsidated by approximately 2000 units of the coat protein $(\mathrm{CP})$ in elongated and flexuous virions. This genomic RNA encodes a large polyprotein that is processed in about 10 gene products in a cascade of proteolytic processing by three virus-encoded proteases: P1, helper-component protease (HC-Pro) and nuclear inclusion $a$ protease (NIaPro) (Revers and García 2015). An additional gene product, P3NPIPO, results from a transcription slippage mechanism (Rodamilans et al. 2015).

In a cucurbit survey in 2013 in Huerta de Vera, Valencia (Spain), we found two different potyviruses co-infecting plants of several cultivars of two cucurbit species, zucchini (Cucurbita pepo L.) and melon (Cucumis melo L.). One of the potyviruses was Zucchini yellow mosaic virus (ZYMV) and the other WMV. While the Vera isolate of ZYMV induced severe symptoms in zucchini or melon plants (Cordero et al. 2017a), that of WMV only induced mild symptoms and infections were not easy to visually diagnose. Mild symptoms are not uncommon in isolates of this viral species (Juarez et al. 2013), which makes difficult to manage the disease in the field, as well as tracking infection in breeding programs aimed to seek for genetic resistance.

Recently, we have introduced a new generation of markers to track plant virus infection, based on the production of plant endogenous pigments that are readily visible to the naked human eye in the infected tissues. These pigments accumulate in those tissues were the viruses express their genomes and replicate. Plant virus-mediated expression of the Antirrhinum majus transcription factor Rosea1 induces accumulation of reddish anthocyanins in the infected tissues (Bedoya et al. 2012). Rosea1 is a MYB R2R3-type transcription factor that, in a cell-autonomous manner, activates the production of companion regulatory proteins, bHLH and WD40, and form a ternary complex (MBW) that activates the biosynthesis of colored anthocyanins (Zhang et al. 2014; Passeri et al. 2016). We showed that plant viruses that belong to different genus and families induce a distinctive reddish pigmentation in the infected tissues when they express Rosea1 (Bedoya et al. 2012; Majer et al. 2013; Cordero et al. 2017b). Similarly, infections with plant viruses that express Pantoea ananatis phytoene synthase (crtB), a key enzyme in the carotenoid biosynthesis pathway of this soil bacterium, 
produce the yellow pigmentation of infected tissue (Majer et al. 2017). In this case, tissue pigmentation results from a combination of endogenous carotenoid accumulation and chlorophyll reduction. Notably, melon and other species in genus Cucurbita, along with many other genera of cucurbits, do not accumulate anthocyanins (Paris 2016). Consistently, the red Rosea1 marker did not produce the expected red pigmentation in melon or zucchini plants infected with a ZYMV recombinant clone that expressed this transcription factor (Cordero et al. 2017a). Conversely, carotenoids serve as major pigments of plant and fruit tissues in many cucurbits, whose color is determined by a combination of chlorophyll and carotenoid pigments (Gur et al. 2016). In fact, the yellow crtB marker produced a bright yellow pigmentation of zucchini tissues infected with ZYMV, suggesting that, in cucurbits, this is the visual marker or choice (Majer et al. 2017). To facilitate tracking the infection of cucurbits by WMV in breeding programs, particularly in the case of difficult isolates that produce mild symptoms, in this work we aimed to build an infectious clone of the Vera isolate of WMV, to tag it with the yellow crtB visual marker and to analyze the outcome of inoculations in different cucurbit species.

To build an infectious clone of the Vera isolate of WMV, we initially amplified an internal cDNA fragment of the viral genome by reverse transcription (RT) followed by the polymerase chain reaction (PCR) using as a template an RNA preparation from an infected melon plant harvested in 2013 in Huerta de Vera (Valencia, Spain). Primers used in this initial RT-PCR were based on the sequence of the reference WMV sequence variant NC_006262.1 and were designed to amplify an approximately $1000 \mathrm{bp} \mathrm{PCR}$ product at around the two thirds length of the viral genome. The sequences of all primers used in this work are in Supplementary Table S1). The PCR product was sequenced and a BLAST analysis (Altschul et al. 1990) indicated that the Vera isolate of WMV was closely related (99\% identity) to sequence variant EU660581.1. A new set of primers based on this sequence variant was designed to amplify a second fragment of the WMV genome at around the one thirds length of the viral genome. Finally, the terminal 5' and 3' ends of the WMV sequence were obtained by rapid amplification of cDNA ends (RACE) (Schaefer 1995). On the basis of all these experimental sequences, we designed primers to amplify the whole WMV genome in three cDNA fragments and to assemble them into the binary plasmid pG35Z (López-González et al. 2017; Cordero et al. 2017a) that contains the Cauliflower mosaic virus (CaMV) 35S promoter and terminator. A plasmid (pGWMV-Vera) that contained the whole WMV genome was selected and sequenced. The sequence of the selected WMV clone was deposited in GenBank as the Vera isolate of WMV (WMV-Vera; GenBank accession number MH469650). A 
BLAST analysis with the whole viral genome confirmed previous result obtained with a partial fragment. The WMV-Vera showed the highest similarity $(99.5 \%)$ with the FMF00LL1 isolate of WMV (EU660581.1), collected in France in year 2000 (Desbiez and Lecoq 2008).

We planned to insert the $P$. ananatis crtB cDNA between NIb and $\mathrm{CP}$ cistrons of WMV. To facilitate the releasing of this recombinant protein from the viral polyprotein, the cDNA was flanked with sequences to complement the split NIb/CP proteolytic site. While at the nucleotide level we chose sequences not present in WMV to avoid undesired recombination during virus replication, at the amino acid level sequences of both proteolytic sites (NIb/crtB and crtB/CP) exactly matched the native $-8 /+3$ sequence of NIb/CP (Fig. 1a). For this manipulation, we subcloned the BamH I-Apa I fragment of pGWMV-Vera in a basic cloning vector, inserted the crtB cDNA including the flanking sequences by Gibson assembly (Gibson et al. 2009) and re-inserted the modified fragment into pGWMV-Vera to obtain pGWMV-crtB. The sequence of the corresponding recombinant clone was also deposited in GenBank (WMV-crtB; GenBank accession number MH46965).

To analyze the infectivity of both WMV clones, we transformed Agrobacterium tumefaciens $\mathrm{C} 58 \mathrm{C} 1$, harboring the helper plasmid pCLEAN-S48 (Thole et al. 2007), and agroinoculated Nicotiana benthamiana and melon (cultivar Piñonet Piel de Sapo) plants. All agroinoculated plants started to show symptoms of infection at approximately one week after inoculation. Symptoms were mild, and particularly in the case of melon plants very difficult to visually diagnose. This agreed on previous observations with the Vera isolate of WMV, which definitively produces mild symptoms. Interestingly, in contrast to plants inoculated with WMV-Vera, in plants inoculated with WMV-crtB, symptomatic tissues soon turned yellow, highlighting infected tissue (Fig. $1 \mathrm{~b}$ and c). These results indicated that the crtB marker facilitates the visual diagnosis of a mild WMV isolate, such as Vera.

Next, we investigated how general this property was and whether WMV-crtB could be a useful tool in cucurbits breeding programs. To this aim, we agroinoculated sets of 5 plants of three melon and three Cucurbita spp. accessions commonly used in breeding programs with WMV-Vera and WMV-crtB. In the case of melon, we chose two commercial cultivars, Piñonet Piel de Sapo and Vedrantais, both susceptible to WMV and belonging to the two melon groups most appreciated in the international markets (Group Ibericus subgroup Piel de Sapo and group Cantalupensis subgroup Charentais, respectively) (Pitrat 2016), and one exotic Indian melon, PI 414723, belonging to the Momordica group (Pitrat 2016), also known as snapmelons. This accession was reported as resistant to WMV, displaying an initial mosaic 
and a further recovery (Gilbert et al. 1994). In the case of Cucurbita spp., we chose the zucchini cultivar MU-CU-16 of C. pepo (Formisano et al. 2012) and the pumpkin cultivar PI604506 (Large Cheese) of C. moschata, both susceptible to WMV. The African pumpkin accession Nigerian Local, reported to be resistant to potyvirus (Brown et al. 2003), was included as resistant control.

After inoculation, we started to visually score infection symptoms in the different groups of plants, using an arbitrary scale from 0 (symptomless) to 4 (severe symptoms) at different days post-inoculation (dpi). The average symptom scores at 7,15 and 30 days are represented in Fig. 2 (see full data in Supplementary Table S2). In average, the insertion of the crtB marker (dashed lines) increased the symptom scores of the two highly susceptible melon cultivars Piñonet Piel de Sapo and Vedrantais at early time post-inoculation (7 and 15 dpi). This difference mostly disappeared at a longer time (30 dpi) (Fig. 2a). No meaningful difference was observed in the less susceptible snapmelon accession (PI 414723) and in all assayed Cucurbita spp. accessions (Fig. 2a and b). At 30 dpi, WMV was diagnosed by Western blot analysis using an anti-CP antibody cross-linked to alkaline phosphatase. All agroinoculated plants that survived the experiments from the two susceptible melon (Piñonet Piel de Sapo and Vedrantais) and Cucurbita spp. (zucchini MU-CU-16 and pumpkin Large Cheese) cultivars were diagnosed as infected with WMV. On the contrary, all assayed plants from the two resistant cultivars (snapmelon PI 414723 and C. moschata Nigerian Local) were diagnosed as free of WMV (Supplementary Table S3).

To collect more unbiased data, we repeated the experiment and measured with a colorimeter the Hue angle color parameter in the first leave above the inoculated at 7 and 15 dpi. Values of Hue angle varied between 129 and 133 in melon and between 129 and 136 in Cucurbita spp. in the spectrum from yellow to green color. ANOVA results indicated significant differences in this color parameter, both at 7 and 15 dpi $(p<0.05)$, with lower values for Hue angles (more yellowish) in leaves of plants inoculated with WMV-crtB (Fig. 3) in most melon accessions and in zucchini (at $7 \mathrm{dpi}$ ). Taken together, these results indicate that the WMV-mediated expression of crtB slightly increase yellowing of infected tissue, what is only visually appreciated in susceptible melon cultivars (Piñonet Piel de Sapo and Vedrantais). In the less susceptible melon genotype (PI 414723) and in zucchini, yellowing is only appreciated by colorimetric measurement.

Finally, we asked what the origin of the yellow pigmentation of the infected tissues was. To learn about this, we measured two colored carotenoids, lutein and $\beta$-carotene, in the two leaves above the inoculated in two plants per treatment (Olives Barba et al. 2006). Fig. 4 
represents the lutein and $\beta$-carotene content in these leaves at 15 dpi. In general, tissues of melon plants accumulated more $\beta$-carotene and less lutein than Cucurbita spp. plants, both under infection of WMV-Vera (average content in $\mathrm{mg} / \mathrm{kg}$ fresh weight: 62.9 vs. 41.9 and 55.8 vs. 70.2, respectively) and WMV-crtB (average content 84.7 vs 73.6 and 68 vs 94), which is consistent with previous studies of pigment profiles performed in these species (Qin et al. 2011; Cuevas et al. 2009; Azevedo-Meleiro and Rodriguez-Amaya 2007). An increase in the accumulation of these two colored carotenoids was observed in all susceptible accessions when they were infected with WMV-crtB compared to controls infected with WMV-Vera. However, while in melon cultivars both pigments have a similar increase in the susceptible zucchini and pumpkin cultivars the main increment was due to the orange pigment $\beta$-carotene $(p<0.05)$ (Fig. 4). The different effect of the viral-based expression of crtB in susceptible melon and Cucurbita spp. cultivars likely explains why the visual yellow marker works better in melon. The orange colored $\beta$-carotene is masked in the greener Cucurbita tissues.

In conclusion, the results of this work indicate that the recombinant WMV-crtB clone is highly infectious in cucurbits and, in susceptible melon cultivars, produces a temporary light yellow pigmentation of the infected tissues that facilitates the early diagnosis of the infection by simple visual inspection. However, in susceptible cultivars of Cucurbita spp., yellow pigmentation was not so clearly appreciated. Analysis of carotenoid accumulation in infected tissues suggested that this difference results from a different ratio of lutein to $\beta$ carotene accumulation in both genera. Therefore, the recombinant clone WMV-crtB may be a useful tool to track WMV resistance by visual diagnosis in melon breeding programs.

Compliance with ethical standards. The authors confirm that this work complies the Ethical Rules applicable for this journal.

Ethical approval. This work does not contain any study with humans or animals.

Conflict of interest. The authors declare that they have no conflict of interest.

Funding. This research was supported by grants BIO2014-54269-R, AGL2014-53398-C2-2R, BIO2017-83184-R, and AGL2017-85563-C2-1-R from the Spanish Ministerio de Economía, Industria y Competividad (co-financed FEDER funds). 


\section{References}

Altschul, S. F., Gish, W., Miller, W., Myers, E. W., \& Lipman, D. J. (1990). Basic local alignment search tool. Journal of Molecular Biology, 215(3), 403-410.

Azevedo-Meleiro, C. H., \& Rodriguez-Amaya, D. B. (2007). Qualitative and Quantitative Differences in Carotenoid Composition Among Cucurbita moschata, Cucurbita maxima, and Cucurbita pepo. Journal of agricultural and food chemistry, 55(10), 4027-4033.

Bedoya, L. C., Martínez, F., Orzáez, D., \& Daròs, J. A. (2012). Visual tracking of plant virus infection and movement using a reporter MYB transcription factor that activates anthocyanin biosynthesis. Plant Physiol., 158(3), 1130-1138.

Brown, R. N., Bolanos-Herrera, A., Myers, J. R., \& Jahn, M. M. (2003). Inheritance of resistance to four cucurbit viruses in Cucurbita moschata. Euphytica, 129(3), 253-258.

Cordero, T., Cerdán, L., Carbonell, A., Katsarou, K., Kalantidis, K., \& Daròs, J. A. (2017a). Dicer-Like 4 Is Involved in Restricting the Systemic Movement of Zucchini yellow mosaic virus in Nicotiana benthamiana. Molecular plant-microbe interactions : MPMI, 30(1), 63-71.

Cordero, T., Mohamed, M. A., López-Moya, J. J., \& Daròs, J. A. (2017b). A recombinant Potato virus $Y$ infectious clone tagged with the Roseal visual marker (PVY-Ros1) facilitates the analysis of viral infectivity and allows the production of large amounts of anthocyanins in Plants. Frontiers in microbiology, 8, 611.

Cuevas, H. E., Staub, J. E., Simon, P. W., \& Zalapa, J. E. (2009). A consensus linkage map identifies genomic regions controlling fruit maturity and beta-carotene-associated flesh color in melon (Cucumis melo L.). TAG. Theoretical and applied genetics. Theoretische und angewandte Genetik, 119(4), 741-756.

Desbiez, C., \& Lecoq, H. (2004). The nucleotide sequence of Watermelon mosaic virus (WMV, Potyvirus) reveals interspecific recombination between two related potyviruses in the 5' part of the genome. Archives of Virology, 149(8), 1619-1632.

Desbiez, C., \& Lecoq, H. (2008). Evidence for multiple intraspecific recombinants in natural populations of Watermelon mosaic virus (WMV, Potyvirus). Archives of Virology, 153(9), 1749-1754.

Formisano, G., Roig, C., Esteras, C., Ercolano, M. R., Nuez, F., Monforte, A. J., et al. (2012). Genetic diversity of Spanish Cucurbita pepo landraces: an unexploited resource for summer squash breeding. Genetic Resources and Crop Evolution, 59(6), 1169-1184. 
Gibson, D. G., Young, L., Chuang, R. Y., Venter, J. C., Hutchison, C. A., 3rd, \& Smith, H. O. (2009). Enzymatic assembly of DNA molecules up to several hundred kilobases. Nat. Methods, 6(5), 343-345.

Gilbert, R. Z., Kyle, M. M., Munger, H. M., \& Gray, S. M. (1994). Inheritance of Resistance to Watermelon Mosaic Virus in Cucumis melo L. Hortscience, 29(2), 107-110.

Gur, A., Gonda, I., Portnoy, V., Tzuri, G., Chayut, N., Cohen, S., et al. (2016). Genomic Aspects of Melon Fruit Quality. In R. Grumet, N. Katzir and J. García-Mas (Eds.), Genetics and Genomics of the Cucurbitaceae (pp. 377-408), Springer International Publishing AG 2016.

Juarez, M., Legua, P., Mengual, C. M., Kassem, M. A., Sempere, R. N., Gómez, P., et al. (2013). Relative incidence, spatial distribution and genetic diversity of cucurbit viruses in eastern Spain. Annals of Applied Biology, 162(3), 362-370.

López-González, S., Aragonés, V., Daròs, J. A., Sánchez, F., \& Ponz, F. (2017). An infectious cDNA clone of a radish-infecting Turnip mosaic virus strain. European Journal of Plant Pathology, 148(1), 207-211.

Majer, E., Daròs, J. A., \& Zwart, M. P. (2013). Stability and fitness impact of the visually discernible Rosea1 marker in the Tobacco etch virus genome. Viruses, 5(9), 21532168 .

Majer, E., Llorente, B., Rodríguez-Concepción, M., \& Daròs, J. A. (2017). Rewiring carotenoid biosynthesis in plants using a viral vector. Scientific reports, 7, 41645.

Olives Barba, A. I., Cámara Hurtado, M., Sánchez Mata, M. C., Fernández Ruiz, V., \& López Sáenz de Tejada, M. (2006). Application of a UV-vis detection-HPLC method for a rapid determination of lycopene and $\beta$-carotene in vegetables. Food chemistry, 95(2), 328-336.

Ouibrahim, L., Mazier, M., Estevan, J., Pagny, G., Decroocq, V., Desbiez, C., et al. (2014). Cloning of the Arabidopsis rwml gene for resistance to Watermelon mosaic virus points to a new function for natural virus resistance genes. The Plant Journal, 79(5), 705-716.

Paris, H. S. (2016). Genetic Resources of Pumpkins and Squash, Cucurbita spp.. In R. Grumet, N. Katzir y J. García-Mas (eds.), Genetics and Genomics of the Cucurbitaceae (pp. 111-154), Springer International Publishing AG 2016.

Passeri, V., Koes, R., \& Quattrocchio, F. M. (2016). New challenges for the design of high value plant products: stabilization of anthocyanins in plant vacuoles. Frontiers in plant science, 7, 153. 
Pitrat, M. (2016). Melon Genetic Resources: Phenotypic Diversity and Horticultural Taxonomy. In R. Grumet, N. Katzir y J. García-Mas (eds.), Genetics and Genomics of the Cucurbitaceae (pp. 25-60), Springer International Publishing AG 2016.

Qin, X., Coku, A., Inoue, K., \& Tian, L. (2011). Expression, subcellular localization, and cisregulatory structure of duplicated phytoene synthase genes in melon (Cucumis melo L.). Planta, 234(4), 737-748.

Revers, F., \& García, J. A. (2015). Molecular biology of potyviruses. Adv. Virus Res., 92, 101-199.

Rodamilans, B., Valli, A., Mingot, A., San León, D., Baulcombe, D., López-Moya, J. J., et al. (2015). RNA polymerase slippage as a mechanism for the production of frameshift gene products in plant viruses of the Potyviridae family. Journal of Virology, 89(13), 6965-6967.

Schaefer, B. C. (1995). Revolutions in rapid amplification of cDNA ends: new strategies for polymerase chain-reaction cloning of full-length cDNA ends. Analytical Biochemistry, 227(2), 255-273.

Thole, V., Worland, B., Snape, J. W., \& Vain, P. (2007). The pCLEAN dual binary vector system for Agrobacterium-mediated plant transformation. Plant Physiol., 145(4), 1211-1219.

Zhang, Y., Butelli, E., \& Martin, C. (2014). Engineering anthocyanin biosynthesis in plants. Current Opinion in Plant Biology, 19, 81-90. 


\section{FIGURES}
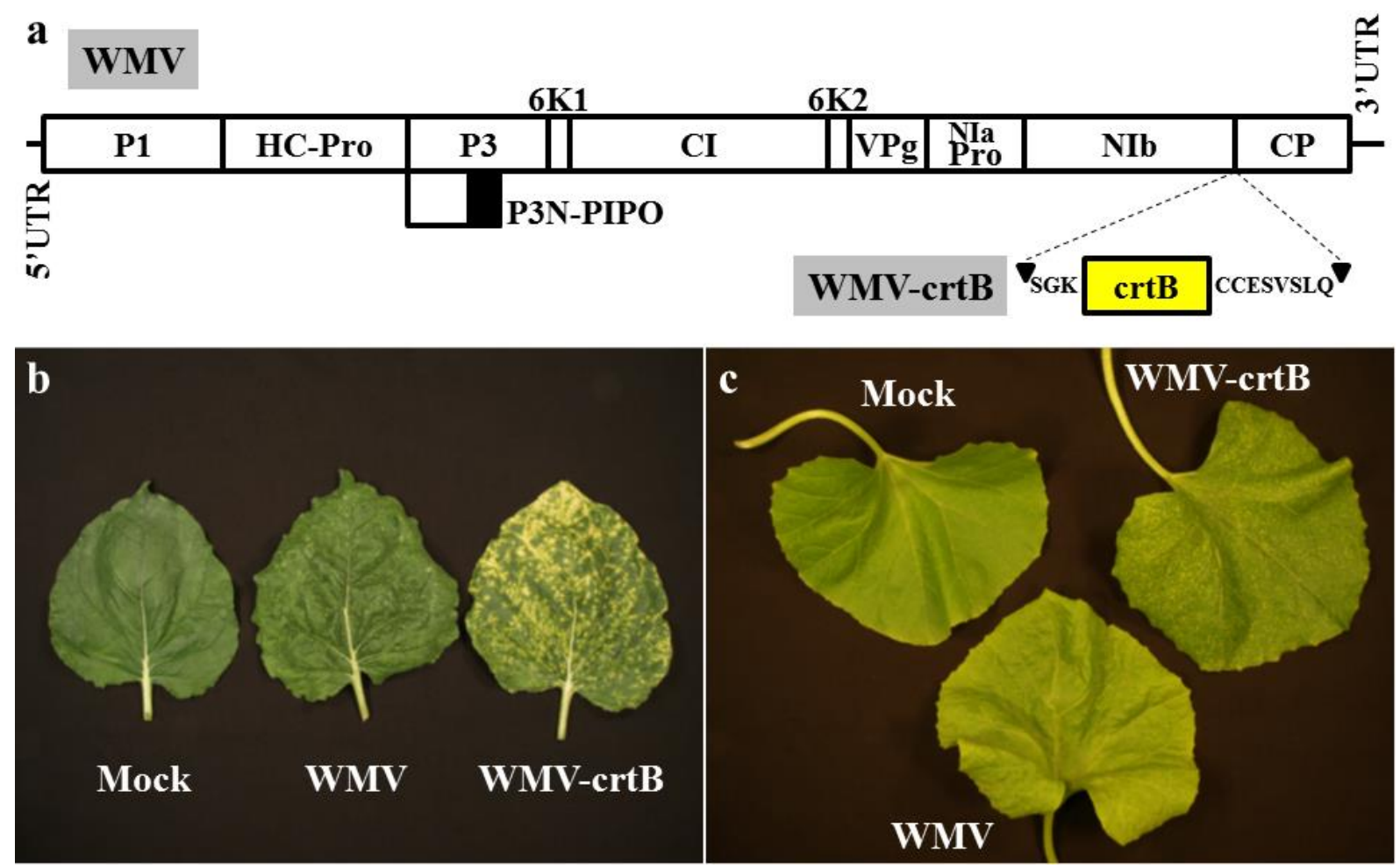

Fig. 1. Symptoms of WMV-Vera and WMV-crtB on infected $N$. benthamiana and Piñonet Piel de Sapo melon plants. (a) Schematic representation of WMV-Vera and WMV-crtB. Lines represent the virus 5' and 3' untranslated regions (UTR) and boxes the different viral cistrons (P1, HC-Pro, P3, P3N-PIPO, 6K1, CI, 6K2, VPg, NIaPro, NIb and CP) as indicated. crtB is indicated by a yellow rectangle. Inserted amino acid sequences that flanked crtB and complement the split NIb/CP proteolytic site are indicated. (b and c) Representative upper non-inoculated leaves from (b) N. benthamiana and (c) Piel de Sapo melon plants mockinoculated or agroinoculated with WMV-Vera or WMV-crtB, as indicated. Pictures were taken 28 dpi. 

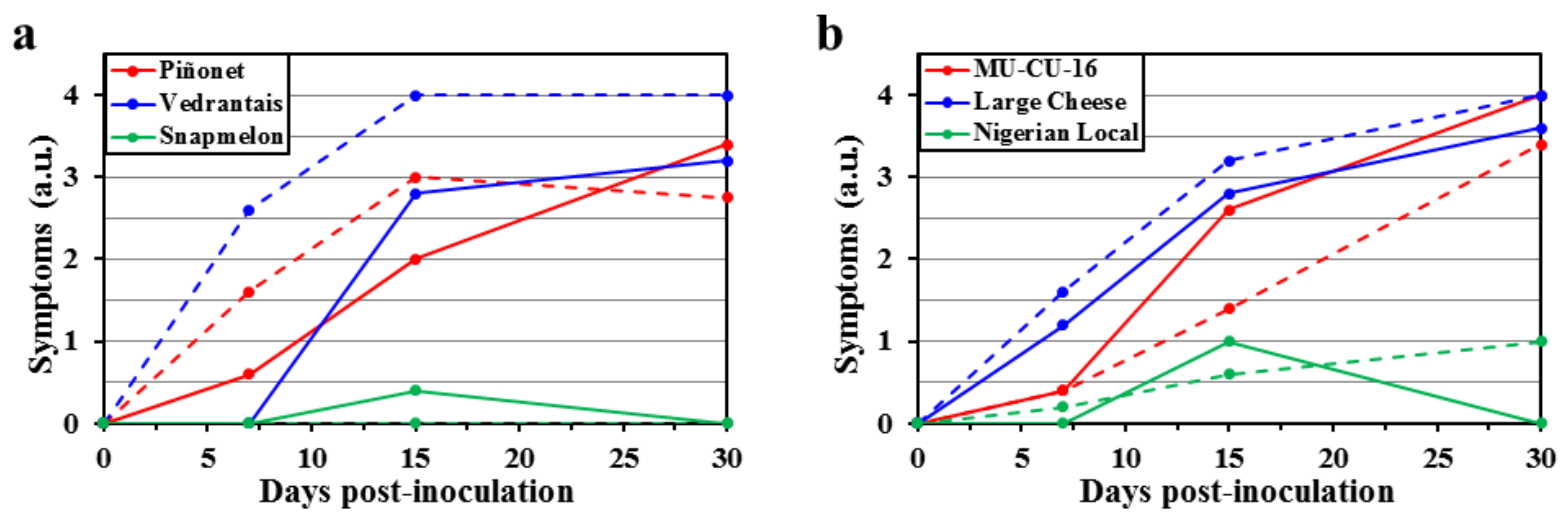

Fig. 2. Graphic representation of WMV-Vera (solid lines) and WMV-crtB (dashed lines) infection symptoms in a series of (a) melon and (b) Cucurbita spp. cultivars as indicated. Symptoms were visually scored at different dpi in an arbitrary scale from 0 (symptomless) to 4 (severe symptoms). The average value of 5 replicate plants is represented. 

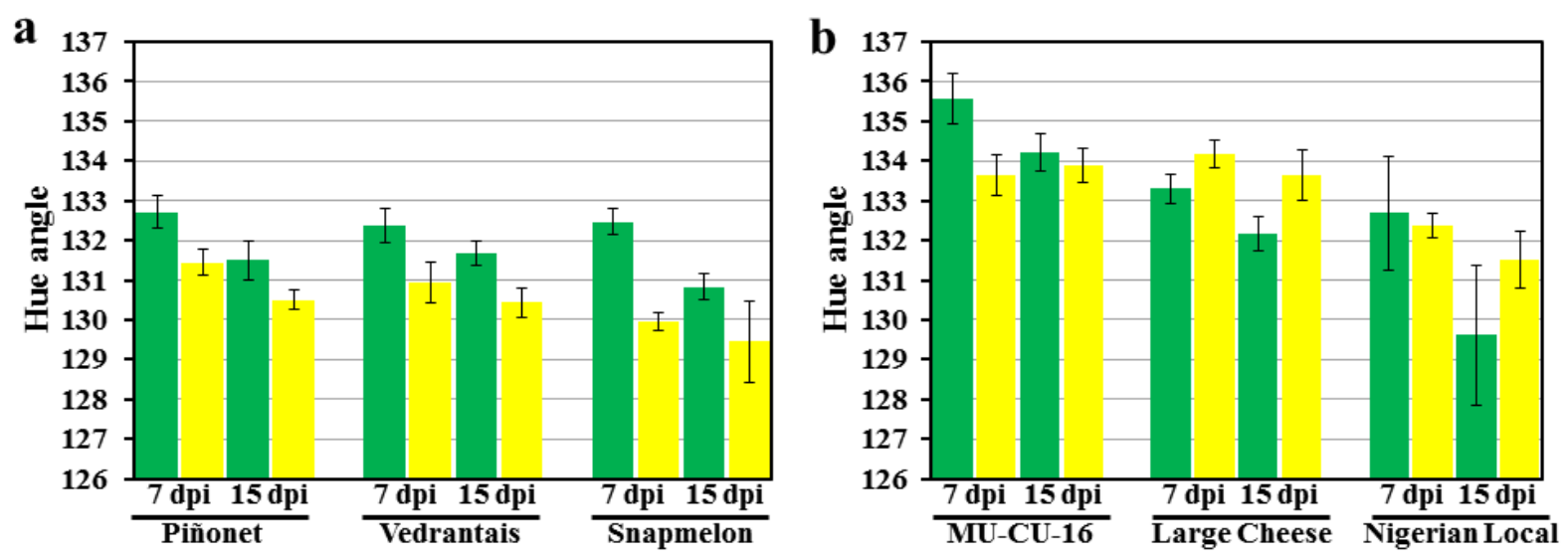

Fig. 3. Graphic representation of the Hue angle measured in leaves of (a) melon and (b) Cucurbita spp. cultivars, as indicated, inoculated with WMV-Vera (green bars) or WMV-crtB (yellow bars) at 7 and 15 dpi. Error bars represent the standard error of 5 replicates plant. 

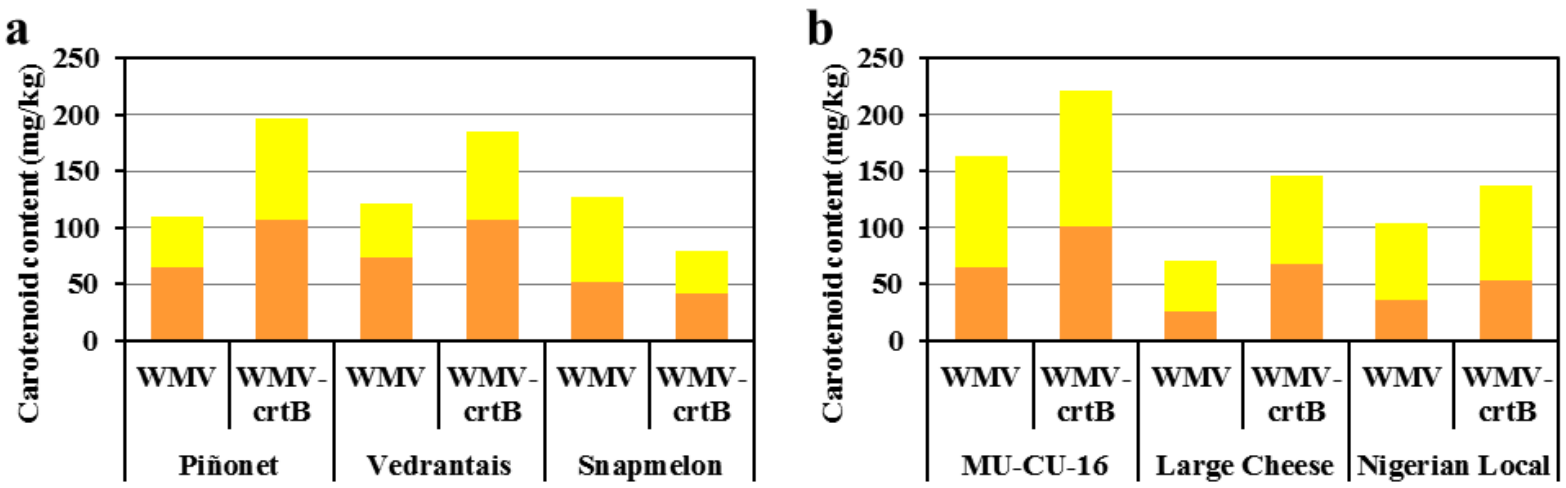

Fig. 4. Graphic representation of the average lutein (yellow bars) and $\beta$-carotene (orange bars) content ( $\mathrm{mg} / \mathrm{kg}$ of fresh weight) measured in the two first leaves (two plants per treatment) at 15 dpi in different (a) melon and (b) Cucurbita spp. Cultivars infected with WMV or WMVcrtB, as indicated. 
Supplementary Table S1. PCR primers used to build the infectious viral clones WMV-Vera and WMV-crtB.

\begin{tabular}{|c|c|c|}
\hline D1448 & 5' -CCGTTTCAAATCCGTCAGTAAATGG-3' & RT first cDNA at $2 / 3^{\text {rd }}$ genome length \\
\hline D1449 & $5^{\prime}-$ AGGGCGTGAAGTGTATGCTGATGAC-3' & \multirow{2}{*}{ PCR first cDNA at $2 / 3^{\text {rd }}$ genome length } \\
\hline D1450 & 5'-AAGAAATTCTTCTCTGAATCATTAG-3' & \\
\hline D1467 & 5'-GTCCCGTCATGCTCTTTGCTACGGG-3' & RT cDNA at $1 / 3^{\text {rd }}$ genome length \\
\hline D1468 & $5^{\prime}$-GGTGTAGCGCAACAAAGGATGAAG-3' & \multirow{2}{*}{ PCR cDNA at $1 / 3^{\text {rd }}$ genome length } \\
\hline D1469 & 5'-GCAATTCAGGTCTAACTTTCTTCAC-3' & \\
\hline D1502 & $5^{\prime}$-TTAATGGCTCTCCTAAGAGACG-3' & \multirow{3}{*}{ 5' RACE } \\
\hline D1503 & $5^{\prime}-$ TTGTATATGCGCTCAGGCCCGC-3' & \\
\hline D1504 & $5^{\prime}$-GCACTTTGCTGTGCACCCTACTG-3' & \\
\hline D1505 & $5^{\prime}-$ TGAGAGGCACACTGCAAGGGAC-3' & \multirow{2}{*}{ 3' RACE } \\
\hline D1506 & 5' -GTAAATCAGAATATGCATACTTTG-3' & \\
\hline D1553 & $5^{\prime}$-AGGACAACAAACATTACCGTACCTC-3' & RT Gibson 3' fragment \\
\hline D1911 & $\begin{array}{l}\text { 5'-ggaagttcatttcatttggaGAGGAAATTAAAACA } \\
\text { ACTCATAAAG-3' }\end{array}$ & \multirow[t]{2}{*}{ 5' cDNA fragment for Gibson assembly } \\
\hline D1912 & 5'-caattgccatGTTATAGAAGATTTTTCCAACC-3' & \\
\hline D1913 & $5^{\prime}$-cttctataacATGGCAATTGAAAAGGTTTAC-3' & \multirow{2}{*}{$\begin{array}{l}\text { Central cDNA fragment for Gibson } \\
\text { assembly }\end{array}$} \\
\hline D1914 & 5' -aaagtagaaaTGCCACTATAATCTCTGAGTC-3' & \\
\hline D1915 & 5'-tatagtggcaTTTCTACTTTGATTTGCCAAC-3' & \multirow{2}{*}{$\begin{array}{l}\text { 3' cDNA fragment for Gibson } \\
\text { assembly, including a } 50 \text {-nt-long polyA } \\
\text { tail }\end{array}$} \\
\hline D1916 & $\begin{array}{l}5^{\prime} \text {-gttttttttttttttttttttttttttttttttt } \\
\text { tttttttttttttTTTTAGGACAACAAACATTACC-3' }\end{array}$ & \\
\hline
\end{tabular}


Supplementary Table S2. Symptoms of infection visually scored in different melon and Cucurbita spp. cultivars agroinoculated with WMV-Vera and WMV-crtB. Symptoms were scored in five independent plants at different dpi in an arbitrary scale from 0 (symptomless) to 4 (severe symptoms).

\begin{tabular}{|c|c|c|c|c|c|c|}
\hline & \multicolumn{3}{|c|}{ WMV-Vera } & \multicolumn{3}{|c|}{ WMV-crtB } \\
\hline Plant & $\mathbf{7}$ dpi & 15 dpi & 30 dpi & 7 dpi & 15 dpi & 30 dpi \\
\hline Cucumis melo Piel de Sapo Piñonet \\
\hline 1 & 0 & 0 & 4 & 0 & 3 & 2 \\
\hline 2 & 3 & 4 & 4 & 0 & n.d. & n.d. \\
\hline 3 & 0 & 4 & 4 & 4 & 4 & 4 \\
\hline 4 & 0 & 0 & 2 & 4 & 4 & 4 \\
\hline 5 & 0 & 2 & 3 & 0 & 1 & 1 \\
\hline Cucumis melo Charentais Vedrantais \\
\hline 1 & 0 & 4 & 4 & 2 & 4 & 4 \\
\hline 2 & 0 & 0 & 1 & 0 & 4 & 4 \\
\hline 3 & 0 & 4 & 4 & 4 & 4 & 4 \\
\hline 4 & 0 & 2 & 4 & 3 & 4 & 4 \\
\hline 5 & 0 & 4 & 3 & 4 & 4 & 4 \\
\hline Cucumis melo Snapmelon (PI 414723) \\
\hline 1 & 0 & 1 & 0 & 0 & 0 & 0 \\
\hline 2 & 0 & 0 & 0 & 0 & 0 & 0 \\
\hline 3 & 0 & 0 & 0 & 0 & 0 & 0 \\
\hline 4 & 0 & 1 & 0 & 0 & 0 & 0 \\
\hline 5 & 0 & 0 & 0 & 0 & n.d. & n.d. \\
\hline Cucurbita pepo Zucchini MU-CU-16 \\
\hline 1 & 0 & 3 & 4 & 1 & 1 & 3 \\
\hline 2 & 1 & 2 & 4 & 0 & 1 & 3 \\
\hline 3 & 0 & 3 & 4 & 1 & 1 & 4 \\
\hline 4 & 1 & 2 & 4 & 0 & 2 & 3 \\
\hline 5 & 0 & 3 & 4 & 0 & 2 & 4 \\
\hline Cucurbita moschata Pumpkin Large Cheese (PI604506) \\
\hline 1 & 1 & 1 & 2 & 1 & 4 & 4 \\
\hline 2 & 0 & 3 & 4 & 2 & 2 & 4 \\
\hline 3 & 2 & 3 & 4 & 1 & 3 & n.d. \\
\hline 4 & 0 & 3 & 4 & 2 & 4 & 4 \\
\hline 5 & 3 & 4 & 4 & 2 & 3 & 4 \\
\hline Cucurbita spp. Nigerian Local & \\
\hline 1 & 0 & 1 & 0 & 0 & 0 & 1 \\
\hline 2 & 0 & 1 & 0 & 0 & 2 & n.d. \\
\hline 3 & 0 & 1 & 0 & 1 & 0 & 1 \\
\hline 4 & 0 & 1 & 0 & 0 & 1 & 1 \\
\hline 5 & 0 & 1 & 0 & 0 & 0 & 1 \\
\hline
\end{tabular}

n.d., non-determined 
Supplementary Table S3. WMV diagnosis of different melon and Cucurbita spp. cultivars agroinoculated with WMV-Vera and WMV-crtB at 30 dpi. WMV CP was detected by Western blot analysis using a specific antibody cross-linked to alkaline phosphatase. For infected plants, the intensity of the luminescent signal is indicated $(+,++$ or +++$)$.

\begin{tabular}{|c|c|c|}
\hline & WMV-Vera & WMV-crtB \\
\hline Plant & 30 dpi & 30 dpi \\
\hline \multicolumn{3}{|c|}{ Cucumis melo Piel de Sapo Piñonet } \\
\hline 1 & +++ & +++ \\
\hline 2 & +++ & n.d. \\
\hline 3 & +++ & +++ \\
\hline 4 & +++ & +++ \\
\hline 5 & +++ & +++ \\
\hline \multicolumn{3}{|c|}{ Cucumis melo Charentais Vedrantais } \\
\hline 1 & +++ & +++ \\
\hline 2 & + & +++ \\
\hline 3 & +++ & +++ \\
\hline 4 & +++ & +++ \\
\hline 5 & +++ & +++ \\
\hline \multicolumn{3}{|c|}{ Cucumis melo Snapmelon (PI 414723) } \\
\hline 1 & - & - \\
\hline 2 & - & - \\
\hline 3 & - & - \\
\hline 4 & - & - \\
\hline 5 & - & n.d. \\
\hline \multicolumn{3}{|c|}{ Cucurbita pepo Zucchini MU-CU-16 } \\
\hline 1 & + & + \\
\hline 2 & + & + \\
\hline 3 & + & + \\
\hline 4 & + & + \\
\hline 5 & + & + \\
\hline \multicolumn{3}{|c|}{$\begin{array}{l}\text { Cucurbita moschata Pumpkin Large } \\
\text { Cheese (PI604506) }\end{array}$} \\
\hline 1 & + & ++ \\
\hline 2 & + & ++ \\
\hline 3 & + & n.d. \\
\hline 4 & + & +++ \\
\hline 5 & ++ & +++ \\
\hline \multicolumn{3}{|c|}{ Cucurbita spp. Nigerian Local } \\
\hline 1 & - & - \\
\hline 2 & - & - \\
\hline 3 & - & - \\
\hline 4 & - & - \\
\hline 5 & - & - \\
\hline
\end{tabular}

n.d., non-determined 